\title{
Silicon carbide neutron detector prototype testing at the JSI TRIGA reactor for enhanced border and ports security
}

\author{
Vladimir Radulović ${ }^{1}$, Klemen Ambrožič ${ }^{1}$, Ivana Capan², Robert Bernat ${ }^{2}$, Zoran Ereš², Željko Pastuović ${ }^{3}$, \\ Adam Sarbutt $^{3}$, Takeshi Ohshima ${ }^{4}$, Yuichi Yamazaki ${ }^{4}$, Takahiro Makino ${ }^{4}$, José Coutinho ${ }^{5}$, Luka Snoj ${ }^{1,6}$ \\ ${ }^{1}$ Jožef Stefan Institute, Slovenia, ${ }^{2}$ Ruđer Bošković Institute, Croatia, ${ }^{3}$ Australian Nuclear Science and \\ Technology Organisation, Australia, ${ }^{4}$ National Institutes for Quantum and Radiological Science and \\ Technology, Japan, ${ }^{5}$ University of Aveiro, Portugal, ${ }^{6}$ University of Ljubljana, Slovenia
}

\begin{abstract}
In 2016, the "E-SiCure" project (standing for "Engineering Silicon Carbide for Border and Port Security"), funded by the NATO Science for Peace and Security Programme was launched. The main objective is to combine theoretical, experimental and applied research towards the development of radiation-hard SiC-based detectors of special nuclear materials (SNM), with the end goal to enhance border and port security barriers. Prototype neutron detectors, configured as $4 \mathrm{H}-\mathrm{SiC}$-based Schottky barrier diodes, were developed for the detection of secondary charged particles (tritons, alphas and lithium atoms) which are the result of thermal neutron reactions on ${ }^{10} \mathrm{~B}$ and ${ }^{6} \mathrm{LiF}$ layers above the surface of the $4 \mathrm{H}-\mathrm{SiC}$ diodes. We designed a stand-alone prototype detection system, consisting of a preamplifier, shaping amplifier and a multichannel analyser operated by a laptop computer, for testing of neutron detector prototypes at the Jožef Stefan Institute (JSI) TRIGA reactor using a broad beam of reactor neutrons. The reverse bias for the detector diode and the power to electronic system were provided by a standalone battery-powered voltage source. The detector functionality was established through measurements using an ${ }^{241} \mathrm{Am}$ alpha particle source. Two dedicated experimental campaigns were performed at the JSI TRIGA reactor. The registered pulse height spectra from the detectors, using both ${ }^{10} \mathrm{~B}$ and ${ }^{6} \mathrm{LiF}$ neutron converting layers, clearly demonstrated the neutron detection abilities of the $\mathrm{SiC}$ detector prototypes. The computed neutron detection sensitivity of the single prototype detectors demonstrates that scaling SiC detectors into larger arrays, of dimensions relevant for border and port radiation detectors, could enable neutron sensitivity levels matching gas-based detector technology.
\end{abstract}

\section{INTRODUCTION}

Increasingly complex risks like geopolitical instability or decentralized terrorism threats, have led to the urge for deploying nuclear screening systems for detection of illicit trafficking of nuclear materials, and from that, to a growing interest in the field of research and development of new radiation detection technologies suitable for homeland security applications [1,2]. Recent progress in the manufacturing of high-quality bulk and epitaxial silicon carbide ( $\mathrm{SiC}$ ) and processing technologies for fabrication of SiC-based electronic devices [3-5], could enable unprecedented opportunities for future SiC-based detection of neutron and alpha particle emissions. As for existing and commonly used gasbased neutron detectors, namely $\mathrm{BF}_{3}$ and ${ }^{3} \mathrm{He}$ detectors, SiC-based devices have the potential to be simultaneously portable, operable at room temperature and radiation hard [6,7]. However, SiC detector technology may offer significant advantages over gas based neutron detectors. SiC is an inert ceramic material, as opposed to $\mathrm{BF}_{3}$ gas which is highly toxic. ${ }^{3} \mathrm{He}$ detectors are currently the most performant, however the worldwide supply of ${ }^{3} \mathrm{He}$ has dwindled. Moreover, as research and 
development of $\mathrm{SiC}$ is driven by a mature and growing industry of power electronics, the application of $\mathrm{SiC}$ as the active detection material could enable significantly more economic neutron detectors.

In 2016, the E-SiCure project was launched, funded by the NATO Science for Peace and Security programme, its main objective being to combine theoretical, experimental and applied research towards the development of radiation-hard SiC-based detectors of special nuclear materials (SNM), and therefore to enhance border and port security barriers. One of the main project activities was the development and testing of prototype detectors based on $\mathrm{SiC}$ and investigating their neutron detection abilities. In the course of the 3-year project, we developed two versions of prototype neutron detectors, configured as $4 \mathrm{H}$-SiC-based Schottky barrier diodes, which in themselves have the ability of detection of energetic charged particles. The diodes are equipped with thin converting layers based on ${ }^{10} \mathrm{~B}$ and ${ }^{6} \mathrm{LiF}$, located either in contact or in close proximity to the diodes, which upon neutron absorption (through $(n, \alpha)$ reactions on ${ }^{10} \mathrm{~B}$ or $(n, t)$ reactions on ${ }^{6} \mathrm{Li}$ ), generate energetic alpha particles, tritons and lithium recoil nuclei.

This paper presents the realization of prototype $\mathrm{SiC}$ neutron detectors and experimental testing of the prototypes carried out at the Jožef Stefan Institute (JSI) using alpha particle sources and a reactor neutron beam from the JSI TRIGA reactor. The aim of the experimental tests was to establish the functionality of the detectors and to demonstrate and quantify their neutron detection ability, in relation to the neutron detection sensitivity of gas-based neutron detectors.

\section{PROTOTYPE NEUTRON DETECTORS AND ACQUISITION SYSTEM}

\subsection{SiC Detectors}

The active part of the detector prototypes consisted of a $4 \mathrm{H}$-SiC Schottky Barrier Diode (SBD), created by growth of silicon carbide onto silicon carbide substrate. The active layer thickness of the detectors ranged from $25 \mu \mathrm{m}$ to $170 \mu \mathrm{m}$. Thin nickel contacts were created on the top and bottom sides of the SBDs, by a process of deposition and sintering in argon atmosphere. The size of the front Ni contact surface defined the active detector surface: $1 \mathrm{~mm} \times 1 \mathrm{~mm}$ in the first version and $2 \mathrm{~mm} \times 2 \mathrm{~mm}$ in the second version. Figure 1 schematically displays the SBD fabrication process.

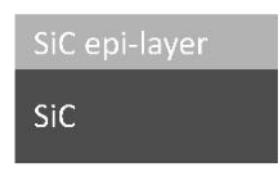

1) SiC epi-layer on SiC

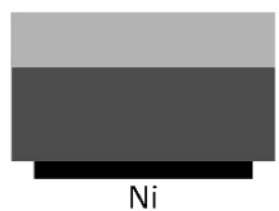

2) Backside Ohmic electrode (deposition and sintering)

$\mathrm{Ni}+950^{\circ} \mathrm{C}, 10 \mathrm{~min}$ in $\mathrm{Ar}$

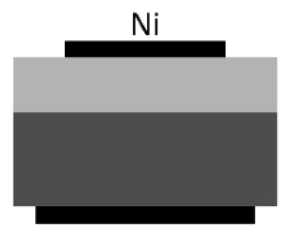

3) Schottky electrode (size: $1 \mathrm{~mm}^{2}$ )

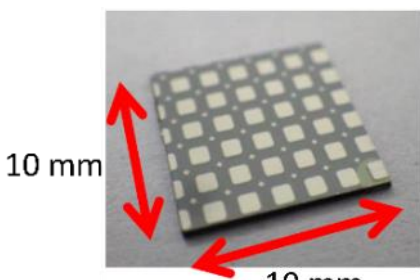

$10 \mathrm{~mm}$

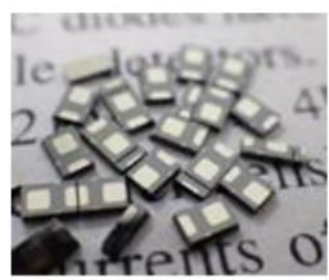

Figure 1: Schematic representation of the manufacture process of 4H-SiC Schottky Barrier Diodes used as neutron detectors.

The SBDs were mounted onto chip carriers with two strip copper contacts. The bottom SBD (Ni) surface was in direct contact with one strip contact, the top SBD Ni surface was wire bonded to the other. Figure 2 displays a close-up photograph of a SiC SBD mounted to a chip carrier. A coaxial cable (connected to the copper contacts on the chip carrier) was used to apply reverse bias voltage to the 
SBD and to convey the signal to the data acquisition system. In order to isolate the detector components under high voltage, the chip carriers bearing the SiC SBDs were mounted into 3D printed polyethylene enclosures.

\subsection{Converting materials}

Due to the very large thermal neutron cross sections of ${ }^{10} \mathrm{~B}$ and ${ }^{6} \mathrm{Li}$ isotopes (around $3843 \mathrm{~b}$ and $938 \mathrm{~b}$ respectively, at an incident neutron energy of $0.0253 \mathrm{eV}[8])$, for the $(n, \alpha)$ and $(n, t)$ reactions, and the fact that in these reactions detectable energetic charged particles are emitted, the chosen converting materials were enriched ${ }^{10} \mathrm{~B}$, ${ }^{6} \mathrm{LiF}$ and ${ }^{10} \mathrm{~B}_{4} \mathrm{C}$. The starting materials were in powder form: pure ${ }^{10} \mathrm{~B},{ }^{10} \mathrm{~B}_{4} \mathrm{C}$ and ${ }^{6} \mathrm{LiF}$.

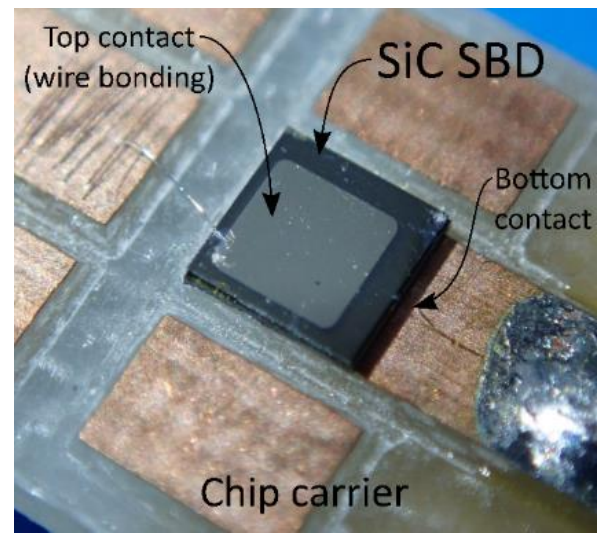

Figure 2: Close-up photograph of a SiC Schottky Barrier Diode (black), mounted and connected to the chip carrier. The light grey square on the diode surface is the top Schottky electrode $(2 \mathrm{~mm} \times 2 \mathrm{~mm})$, defining the active volume of the detector.

\subsection{Data acquisition system}

The data acquisition system used for the measurement of SiC signals consisted of a preamplifier and shaping amplifier sourced from CREMAT, a multichannel analyser sourced from AMPTEK and a laptop computer. In order to minimize the level of electronic noise, power to the system was provided by a standalone power supply, powered by batteries. Reverse bias was applied to the detectors using a high voltage module, also powered by the standalone power supply. Figure 3 displays a schematic representation of the detectors and the data acquisition system components.

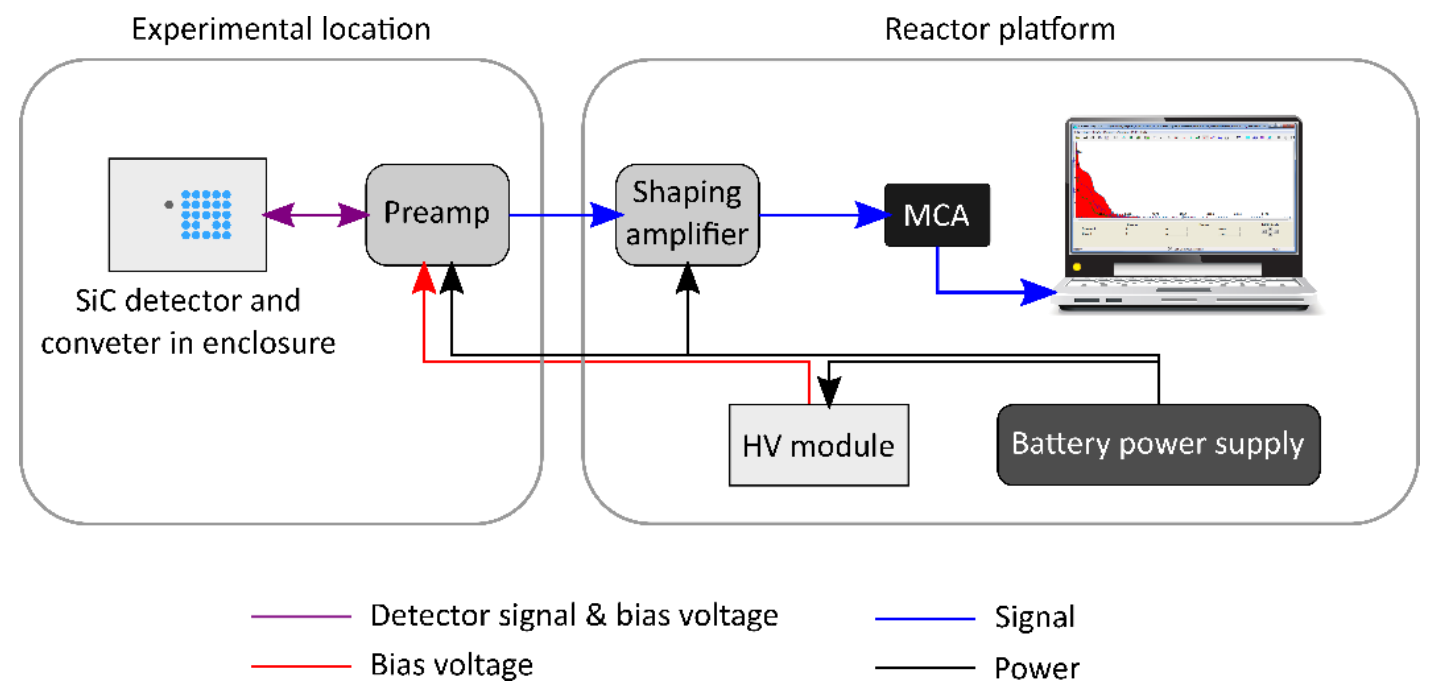

Figure 3: Schematic representation of the data acquisition system. 


\section{EXPERIMENTAL SETUP}

\subsection{JSI TRIGA reactor}

Neutron irradiation testing of the SiC detector prototypes was carried out in the Dry Chamber of the JSI TRIGA reactor. The JSI TRIGA reactor is a $250 \mathrm{~kW}$ TRIGA pool-type research reactor which achieved 1 st criticality in 1966. The reactor is mainly used for research purposes, as a reference neutron irradiation facility for detectors, electronic components and neutron activation analysis, as a test facility for radiation hardness studies in support of specific applications, education and training activities. The Dry Chamber is a large irradiation chamber within the concrete reactor body. It is particularly suited for radiation hardness testing of detectors, electronic components and complete electronic systems [9-11]. Neutrons from the reactor core can be conveyed to the Dry Chamber via a graphite thermalizing column, its cross section being approximately $0.5 \mathrm{~m} \times 0.5 \mathrm{~m}$. The detectors and preamplifiers were installed in the Dry Chamber, signal and power cables were routed to the reactor platform, where the remaining components were located.

\subsection{Version 1 SiC detectors}

In the first version of the prototype detectors, the SiC detectors inside 3D-printed enclosures were mounted in aluminium housings. The 3D-printed enclosures consisted of a base plate and a lid, which had an opening above the SiC SBDs. The aluminium housings also had an opening. Neutron converter layers were made by depositing small quantities of ${ }^{10} \mathrm{~B}$ or ${ }^{6} \mathrm{LiF}$ powder onto adhesive tape. The converter layers were applied to the outside of the 3D printed detector enclosures, above the SBDs, inside the aluminium housings. Figure 4 shows a photograph of two version 1 detectors, assembled and disassembled.

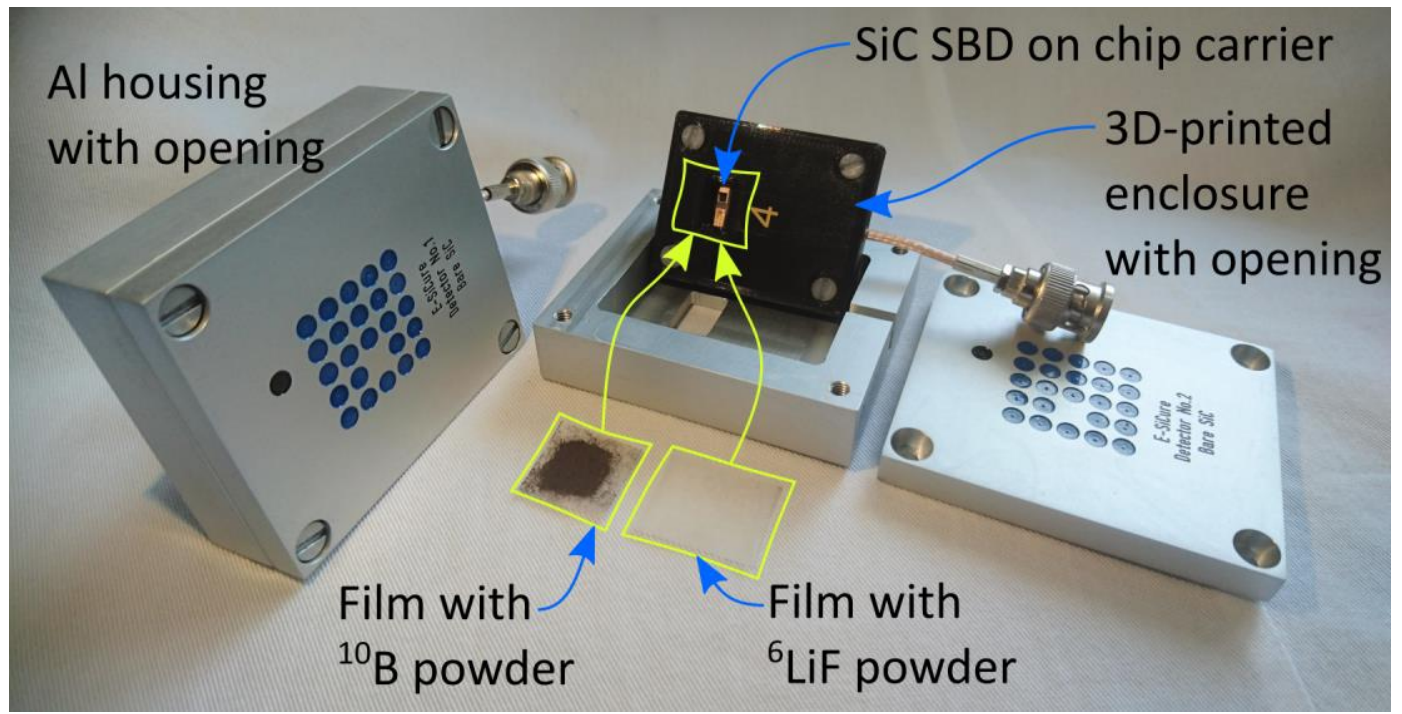

Figure 4: Version $1 \mathrm{SiC}$ detector prototype. Left: assembled detector, right: SiC detector enclosed in 3D-printed enclosure, converting layers, open aluminium housing.

\subsection{Version 2 SiC detectors}

The aim of the second version of prototype detectors was designed with the objective to maximise the attainable neutron detection efficiency. In order to achieve this, several changes were made with respect to version 1 detectors. The SBD area was increased by a factor of 4 ( $2 \mathrm{~mm} \times 2 \mathrm{~mm}$ instead of 1 $\mathrm{mm} \times 1 \mathrm{~mm}$ ). An investigation was carried out on the optimal properties of the neutron converting layer. In the second detector version, converting layers with a deposit of ${ }^{10} \mathrm{~B}$ and ${ }^{6} \mathrm{LiF}$ with a thickness 
of the order of $1 \mu \mathrm{m}$ were used. Commercially available ${ }^{10} \mathrm{~B}_{4} \mathrm{C}$ converting layers on Al substrate, manufactured specifically for neutron detection applications [12] were acquired. ${ }^{6} \mathrm{LiF}$ converter layers of different thicknesses were prepared by thermal evaporation of ${ }^{6} \mathrm{LiF}$ onto Al substrate at the Ruđer Bošković Institute (RBI). Figure 5 displays an atomic force microscopy image and a plot of the profile of one of the realized ${ }^{6} \mathrm{LiF}$ converter layers.
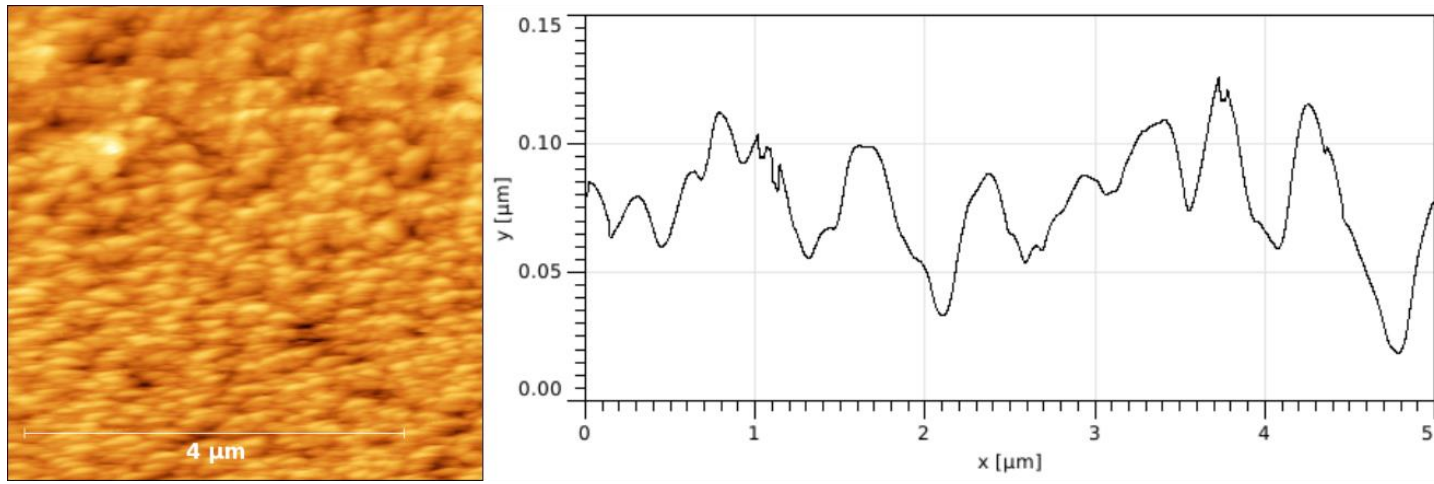

Figure 5: Atomic force microscopy data on one of the ${ }^{6} \mathrm{LiF}$ converter layers obtained by thermal evaporation. Left: AFM image, right: plot of the deposit profile.

Additionally, a series of version 2 detectors was prepared with small quantities of ${ }^{10} \mathrm{~B}_{4} \mathrm{C}$ or ${ }^{6} \mathrm{LiF}$ material deposited directly on the SBD surface. To accomplish this, aqueous solutions of ${ }^{10} \mathrm{~B} 4 \mathrm{C}$ and ${ }^{6} \mathrm{LiF}$ were prepared ( $47 \mathrm{mg}$ of ${ }^{10} \mathrm{~B}_{4} \mathrm{C} / 10 \mathrm{mg}$ of ${ }^{6} \mathrm{LiF}$ dissolved in $4 \mathrm{~mL}$ of $\mathrm{H}_{2} \mathrm{O}$ ). Droplets of around $2.5 \mu \mathrm{L}$ in volume were deposited onto the detector surface and left to dry. Three detectors for each converting material were prepared, with 1, 5 and 15 droplets of solution deposited, respectively.

The third change with respect to the first detector version was the use of significantly larger, vacuumtight enclosures, which contained the detectors and converter layers. The enclosure bodies were manufactured out of stainless steel, with a Plexiglas window. Two height-adjustable support tables in the enclosures allowed precise positioning of the detector and the converter within, and to minimize the distance between the converter layer and the SBD surface (the wire-bonded contact being the main limitation). Figure 6 displays two detector enclosures and neutron converters.

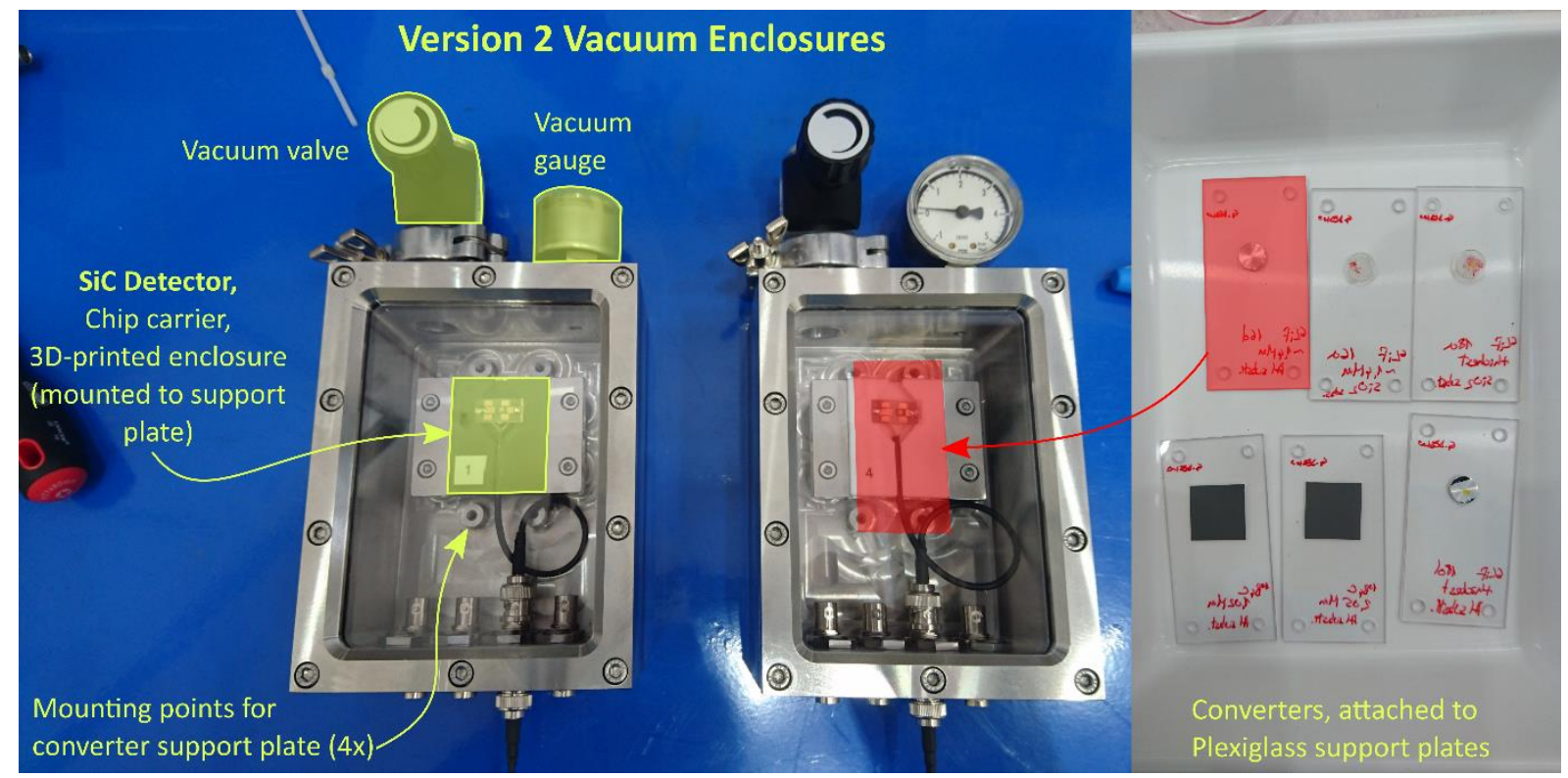

Figure 6: Version 2 detector enclosures. Left: two detector enclosures, right: neutron converting layers attached to Plexiglas support plates. 


\section{RESULTS AND DISCUSSION}

\subsection{Charged particle spectra}

Charged particle spectra originating from neutron interactions in the converting layers were collected with several version 1 and version 2 detectors at different reactor power levels. In all the spectra, we observed a very high peak at low channel numbers, which was attributed to electronic noise, as the peak appeared regardless of whether the detector was irradiated or not. For the same converter material (either ${ }^{10} \mathrm{~B} /{ }^{10} \mathrm{~B}_{4} \mathrm{C}$ or ${ }^{6} \mathrm{LiF}$ ), overall, we observed charged particle spectra with qualitatively similar characteristics. Figure 7 displays charged particle spectra obtained with version 1 and version 2 detectors, using ${ }^{10} \mathrm{~B} /{ }^{10} \mathrm{~B}_{4} \mathrm{C}$ and ${ }^{6} \mathrm{LiF}$ converters.
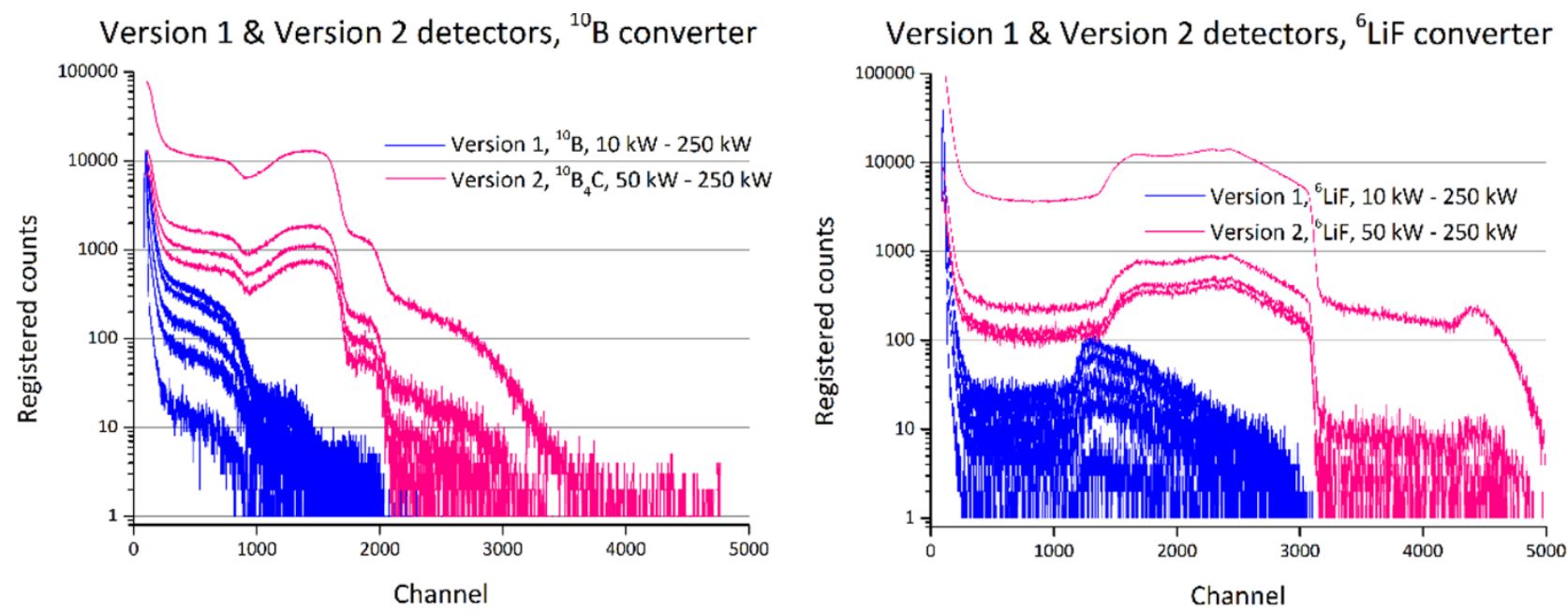

Figure 7: Registered particle spectra from E-SiCure SiC detectors. Version 1 detector spectra marked in blue, Version 2 spectra in pink. Left: measured spectra using ${ }^{10} \mathrm{~B} /{ }^{10} \mathrm{~B}_{4} \mathrm{C}$ converter, right: measured spectra using ${ }^{6}$ LiF converter.

The charged particle spectra obtained with ${ }^{10} \mathrm{~B}$ as converters exhibited four partially overlapping regions which were attributed to the nature and energy of the charged particles originating from ${ }^{10} \mathrm{~B}(\mathrm{n}, \alpha)$ reactions, i.e. $\alpha$ particles and recoil ${ }^{7} \mathrm{Li}$ nuclei. The reaction has two reaction channels with different $Q$ values, and therefore different particle energies:

$$
\begin{aligned}
& { }^{10} \mathrm{~B}+\mathrm{n} \rightarrow \alpha(1776 \mathrm{keV})+{ }^{7} \mathrm{Li}(1013 \mathrm{keV}) ; \mathrm{BR}=6.3 \% ; \mathrm{Q}=2789.5 \mathrm{keV}, \\
& { }^{10} \mathrm{~B}+\mathrm{n} \rightarrow \alpha(1472 \mathrm{keV}){ }^{7} \mathrm{Li} *(840 \mathrm{keV}) ; \mathrm{BR}=93.7 \% ; \mathrm{Q}=2311.9 \mathrm{keV} .
\end{aligned}
$$

In order of increasing channel number (increasing detected charged particle energy), we observe recoil ${ }^{7} \mathrm{Li}$ nuclei from both reaction channels, $\alpha$ particles from both reaction channels, simultaneous detection of a ${ }^{7} \mathrm{Li}$ nucleus and an $\alpha$ particle from the most probable reaction channel (lower charged particle energies) and, finally, simultaneous detection of a ${ }^{7} \mathrm{Li}$ nucleus and $\alpha$ particle from the less probable reaction channel (higher charged particle energies). Significantly better resolution was observed for version 2 detectors, as visible in Figure 7.

The charged particle spectra obtained with ${ }^{6}$ LiF converters and version 1 detectors exhibited a broad peak, attributed to partial energy deposition of tritons and $\alpha$ particles. The ${ }^{6} \mathrm{Li}(\mathrm{n}, \mathrm{t})$ reaction takes place through one channel only:

$$
{ }^{6} \mathrm{Li}+\mathrm{n} \rightarrow \mathrm{t}(2731 \mathrm{keV})+\alpha(2052 \mathrm{keV}) ; \mathrm{Q}=4783 \mathrm{keV} .
$$


Significantly better resolution was observed for version 2 detectors, as the charged particle spectra exhibited two clearly visible broad peaks, attributed to a particles and tritons, respectively. In the recorded spectra from version 2 detectors simultaneous detection events can also be observed.

\subsection{Neutron count rates}

In order to obtain quantitative results allowing the estimation of the detection efficiency of the SiC detectors, a cut-off was applied to the charged particle spectra, allowing for discrimination between the contribution due to electronic noise (and possibly gamma ray interactions) and the contribution due to charged particles, originating from neutron absorption reactions. The cut-off was chosen based on the location and width of the low energy peak attributed to electronic noise. In version 1 detectors, the cut-off was set at channel no. 500, in version 2 at channel no. 250. Measurements were made at several reactor power levels, ranging from $10 \mathrm{~kW}$ (version 1) and $25 \mathrm{~kW}$ (version 2), up to full reactor power, $250 \mathrm{~kW}$. Figure 8 displays the neutron count rates as a function of reactor power. In all the measurements, the number of registered counts was of the order of $10^{4}$, implying uncertainty levels of around $1 \%$ in the measured count rates (impractical to be plotted in the figure). However, the registered neutron count rates do depend also on the chosen energy cut-off in the charged particle spectra. For a conservatively chosen energy cut-off, as in the present case, part of the useful signal (estimated at several $10 \%$ ) is rejected together with the unwanted contribution due to electronic noise. This implies that the "true" neutron count rates are accordingly higher, but of the same order of magnitude as the registered count rates.
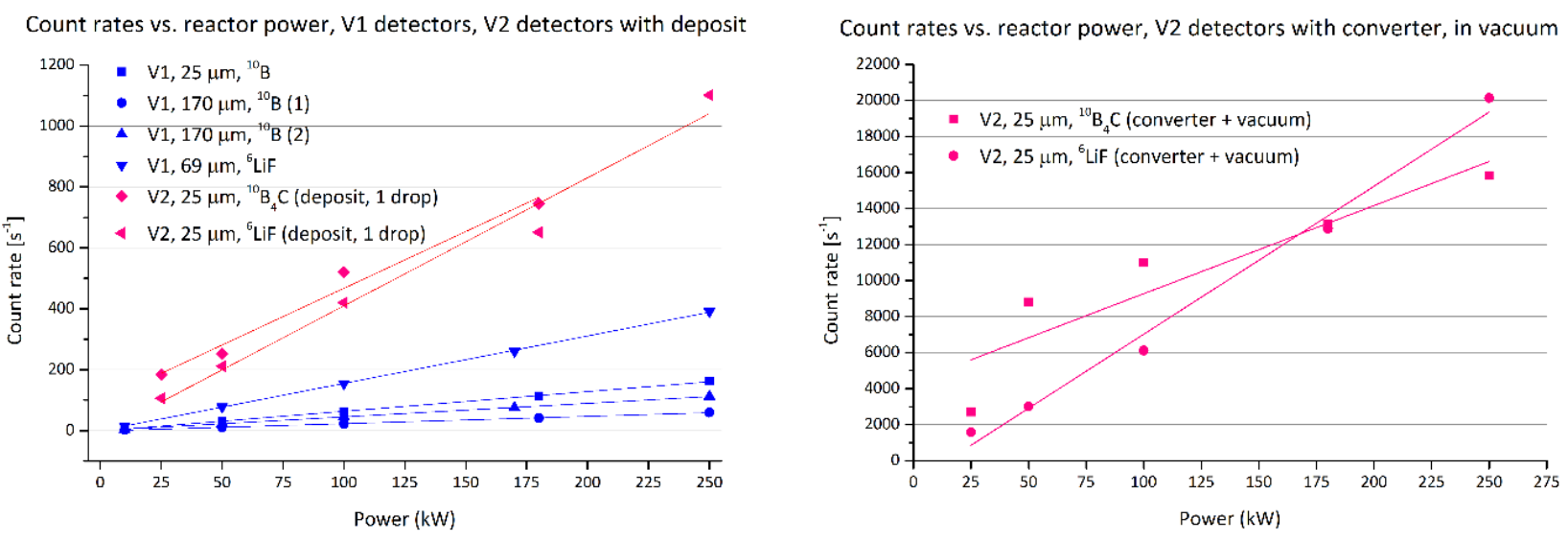

Figure 8: Registered count rates as a function of reactor power for version 1 detectors (in blue) and version 2 detectors (in pink). Left: version 1 and version 2 detectors with converter material deposited on the SBD surface. Right: version 2 detectors with separate converters, in vacuum. The straight lines are linear functions fitted to experimental data.

The neutron count rates are seen to increase linearly with reactor power, except for the version 2 detector with ${ }^{10} \mathrm{~B}_{4} \mathrm{C}$ converter in vacuum, where we observed some deviations, which are at present not understood. More importantly, we observe a very large increase in neutron count rates in version 2 detectors, compared to version 1 . The neutron count rates for version 2 detectors with the smallest amount of deposited material (1 drop of solution) are around two times greater than the most performant version 1 detector. The count rates for version 2 detectors with 5 and 15 drops of solution (not plotted) increased approximately linearly with the amount of deposited material. By far the most performant detectors were the version 2 detectors, with separate converters in vacuum environment (Figure 8, right). The observed count rates for these detectors are around 2 orders of magnitude higher compared to version 1 detectors. The order of magnitude of the neutron flux in the experimental location in the JSI TRIGA Dry Chamber at full reactor power (250 kW) is $10^{7} \mathrm{n} \mathrm{cm}^{-2} \mathrm{~s}^{-1}$ [10], from which 
the neutron detection sensitivities of individual version 1 and version 2 detectors are of the order of $10^{-5}$ and $10^{-3}$ counts $\mathrm{s}^{-1}$ per $\mathrm{n} \mathrm{cm}^{-2} \mathrm{~s}^{-1}$, respectively. Assuming a homogeneous neutron field, increasing the detector surface to typical dimensions of large neutron monitors, i.e. around $0.4 \mathrm{~m}^{2}(2 \mathrm{~m} \times 0.2 \mathrm{~m})$, would enable neutron detection sensitivity values of around $1-10^{2}$, counts s-1 per $\mathrm{cm}^{-2} \mathrm{~s}^{-1}$, which are comparable with neutron detection sensitivity values achievable with gas based detectors.

\section{CONCLUSIONS}

This paper presents the development and testing of SiC SBD neutron detector prototypes and acquisition systems performed in the NATO SPS-funded project E-SiCure, with the motivation to pave the way for the application of SiC detector technology for enhanced border and port security barriers. It is demonstrated that simple detection devices based on SiC SBDs, equipped with neutron to charged particle converter films, based on ${ }^{10} \mathrm{~B}$ and ${ }^{6} \mathrm{Li}$ isotopes, have a clearly measurable neutron response, which varies linearly with the incident neutron flux. The determined detection sensitivity of individual $\mathrm{SiC}$ detectors is such, that scaling to typical dimensions of neutron monitor detectors (i.e. around 50 $\mathrm{cm} \times 2 \mathrm{~m}$ ) would allow comparable performance.

\section{ACKNOWLEDGMENTS}

The present work was financially supported by the NATO Science for Peace and Security Programme, under project no. SPS 985215 - Engineering Silicon Carbide for Border and Port Security - E-SiCure. The JSI project team would like to acknowledge the support from the Slovenian Ministry of Education, Science and Sport (project no. P2-0073 - Reactor Physics). The RBI project team would like to acknowledge financial support from the European Regional Development Fund for the "Center of Excellence for Advanced Materials and Sensing Devices" (Grant No. KK.01.1.1.01.0001). JC acknowledges the Fundaçao para a Ciencia e a Tecnologia (FCT) through project UID/CTM/50025/2013.

\section{REFERENCES}

[1] R. T. Kouzes et al., Neutron detection alternatives to 3 He for national security applications, Nucl. Inst. Meth. A, Vol. 623, Issue 3, pp. 1035-1045, 2010.

[2] A. Wahbi, L. Heng, G. Dercon, Cosmic Ray Neutron Sensing: Estimation of Agricultural Crop Biomass Water Equivalent, Springer, 2018.

[3] T. Kimoto, Material science and device physics in SiC technology for high-voltage power devices, Jpn. J. Appl. Phys. Vol. 54, 040103, 2015.

[4] X. She et al., Review of Silicon Carbide Power Devices and Their Applications, IEEE Transactions on Industrial Electronics, Vol. 64, No. 10, pp. 8193-8205, 2017.

[5] J. Wang et al., IEEE ITRW Working Group Position Paper-System Integration and Application: Silicon Carbide: A Roadmap for Silicon Carbide Adoption in Power Conversion Applications, IEEE Power Electronics Magazine, Vol. 5, No. 2, pp. 40-44, 2018.

[6] W. J. Choyke, H. Matsunami, G. Pensl, Silicon Carbide, Springer, 2004.

[7] T. Kimoto, J. A. Cooper, Fundamentals of Silicon Carbide Technology: Growth, Characterization, Devices, and Applications, Singapore, John Wiley \& Sons, 2014.

[8] R. Capote et al., "Updating and Extending the IRDF-2002 Dosimetry Library," Journal of ASTM International, 9 (4), 2012, pp. 1-9.

[9] V. Radulović et al., "Characterization of ex-core irradiation facilities of the JSI TRIGA Mark II reactor", Proceedings, 21st International Conference Nuclear Energy for New Europe, September 5-7, 2012, Ljubljana, Slovenia.

[10] I. Mandić et al., "Bulk damage in DMILL npn bipolar transistors caused by thermal neutrons versus protons and fast neutrons", IEEE Transactions on Nuclear Science, 51 (4), 2004, pp. 1752-1758. 
[11] I. Mandić, V. Cindro, A. Gorišek, G. Kramberger and M. Mikuž, "Online Integrating Radiation Monitoring System for the ATLAS Detector at the Large Hadron Collider", IEEE Transactions on Nuclear Science, 54 (4), 2007, pp. 1143-1150.

[12] G. Nowak et al., Boron carbide coatings for neutron detection probed by $\mathrm{x}$-rays, ions, and neutrons to determine thin film quality, Journal of Applied Physics, 117, 3, 034901, 2015. 